\title{
Effects of patient movement on measurements of myocardial blood flow and viability in resting ${ }^{15} \mathrm{O}$-water PET studies
}

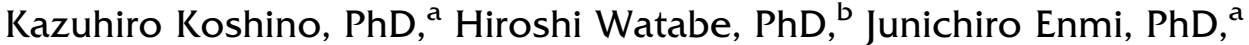 \\ Yoshiyuki Hirano, PhD, ${ }^{a}$ Tsutomu Zeniya, PhD, ${ }^{a}$ Shinji Hasegawa, MD, ${ }^{c}$ \\ Takuya Hayashi, MD, ${ }^{\mathrm{d}}$ Shigeru Miyagawa, MD, ${ }^{\mathrm{e}}$ Yoshiki Sawa, MD, \\ Jun Hatazawa, MD, ${ }^{b}$ and Hidehiro Iida, $\mathrm{DSc}^{\mathrm{a}}$
}

Background. Patient movement has been considered an important source of errors in cardiac PET. This study was aimed at evaluating the effects of such movement on myocardial blood flow (MBF) and perfusable tissue fraction (PTF) measurements in intravenous ${ }^{15} \mathrm{O}$-water PET.

Methods. Nineteen ${ }^{15} \mathrm{O}$-water scans were performed on ten healthy volunteers and three patients with severe cardiac dysfunction under resting conditions. Motions of subjects during scans were estimated by monitoring locations of markers on their chests using an optical motion-tracking device. Each sinogram of the dynamic emission frames was corrected for subject motion. Variation of regional MBF and PTF with and without the motion corrections was evaluated.

Results. In nine scans, motions during ${ }^{15} \mathrm{O}$-water scan (inter-frame (IF) motion) and misalignments relative to the transmission scan (inter-scan (IS) motion) larger than the spatial resolution of the PET scanner $(4.0 \mathrm{~mm})$ were both detected by the optical motion-tracking device. After correction for IF motions, MBF values changed from $0.845 \pm 0.366$ to $0.780 \pm 0.360 \mathrm{~mL} / \mathrm{minute} / \mathrm{g}(P<.05)$. In four scans with only IS motion detected, PTF values changed significantly from $0.465 \pm 0.118$ to $0.504 \pm 0.087 \mathrm{~g} / \mathrm{mL}(P<.05)$, but no significant change was found in MBF values.

Conclusions. This study demonstrates that IF motion during ${ }^{15} \mathrm{O}$-water scan at rest can be source of error in MBF measurement. Furthermore, estimated MBF is less sensitive than PTF values to misalignment between transmission and ${ }^{15} \mathrm{O}$-water emission scans. ( $\mathrm{J}$ Nucl Cardiol 2012;19:524-33.)

Key Words: Myocardial blood flow $\cdot$ water-perfusable tissue fraction $\cdot$ PET $\cdot$ myocardial perfusion imaging $\cdot$ motion correction $\cdot{ }^{15} \mathrm{O}$-labeled water

\section{INTRODUCTION}

Positron emission tomography (PET) has been extensively utilized for a wide range of non-invasive

From the Department of Investigative Radiology, ${ }^{a}$ National Cerebral and Cardiovascular Center Research Institute, Osaka, Japan; Department of Molecular Imaging in Medicine, ${ }^{\mathrm{b}}$ Department of Cardiovascular Surgery, ${ }^{\mathrm{e}}$ Osaka University Graduate School of Medicine, Osaka, Japan; Department of Cardiology, ${ }^{c}$ Osaka Koseinenkin Hospital, Osaka, Japan; Functional Probe Research Laboratory, ${ }^{\mathrm{d}}$ RIKEN Center for Molecular Imaging Science, Hyogo, Japan.

This study was supported by a Research Grant from the New Energy and Industrial Technology Development Organization (NEDO), Japan; a Grant for Translational Research from the Ministry of Health, Labour and Welfare (MHLW) of Japan; a Grant for Advanced Medical Technology from the Ministry of Health, Labour and Welfare (MHLW) of Japan; the Program for Promotion of functional imaging of the myocardium in vivo. When using this method, the global body movements of patients could be a source of quantitative errors. Such

Fundamental Studies in Health Science of the Organization for Pharmaceutical Safety and Research of Japan; and a Grant-in-Aid for Scientific Research from the Ministry of Education, Culture, Sports, Science and Technology (MEXT) of Japan.

Received for publication Apr 5, 2011; final revision accepted Jan 12, 2012.

Reprint requests: Kazuhiro Koshino, PhD, Department of Investigative Radiology, National Cerebral and Cardiovascular Center Research Institute, 5-7-1 Fujishirodai, Suita, Osaka 565-8565, Japan; koshino@ri.ncvc.go.jp.

$1071-3581 / \$ 34.00$

Copyright (c) 2012 The Author(s). This article is published with open access at Springerlink.com

doi:10.1007/s12350-012-9522-0 
movement could be particularly problematic when scans are carried out for a relatively long period. ${ }^{1,2}$ Problems also arise when studies are carried out during physiologically stressed conditions, e.g., a cycling exercise in the PET scanner. ${ }^{3}$ Errors can be attributed not only to the mismatch between the emission and the transmission data but also to the patient motion during each of the emission and/or the transmission scans. ${ }^{3}$

${ }^{15} \mathrm{O}$-water PET studies provide quantitative information regarding myocardial blood flow (MBF) and coronary flow reserve (CFR), as well as a marker of myocardial viability, termed the water perfusable tissue fraction (PTF) or water perfusable tissue index (PTI). ${ }^{4-12}$ The distribution of radioactivity during ${ }^{15} \mathrm{O}$-water PET varies over time; this poses challenges for softwarebased correction of patient movement. Naum et al proposed to correct for such motion based on the rigid body model by aligning two external radioactive markers on the back of each subject. This study was conducted by performing dynamic scans while the subjects were under resting conditions and engaged in a cycling exercise. ${ }^{3}$ Although no correction was made for the misalignment between transmission and emission scans, their study demonstrated reasonable improvement in calculated MBF values.

In our previous work, we developed an alternative system that uses an optical motion-tracking device to detect and correct for the patient's global movement during a cardiac ${ }^{15} \mathrm{O}$-water PET study. ${ }^{13}$ Our system provides a correction for movement during dynamic scanning, as well as for misalignment between the transmission and the emission scans, to compensate for errors in attenuation correction procedures. We evaluated and methodologically validated the inherent accuracy of this system in a cardiac phantom study. The correction for simulated global movement in a ${ }^{15} \mathrm{O}$ water cardiac PET study of a healthy volunteer has also demonstrated reasonable regional MBF values, compared to values not adjusted for movement.

The purpose of this study was to evaluate the effects of global movements of subjects on quantification of MBF and PTF. Our previously validated system was used to detect and correct for the global movements of healthy volunteers, as well as patients who have suffered from severe cardiac dysfunction, during ${ }^{15} \mathrm{O}$-water PET studies under resting conditions.

\section{METHODS}

\section{Subjects}

Subjects consisted of 10 healthy volunteers and 3 patients with previous myocardial infarction. The volunteers were all male, 22-32 years of age (mean \pm 1 standard deviation $(\mathrm{SD})$
$25 \pm 3$ years). The volunteers had no signs or symptoms of ischemic heart disease. Patients were studied before and after the cell transplantation therapy with autologous myoblast sheets (AMS). ${ }^{14,15}$ Scans were carried out by independent clinical research project, but were included in this study by mutual agreement. Two of the patients were male, the other was female; patients were 43-63 years of age. All patients had left ventricular assist systems (LVASs) at the time of PET study, except for one patient who received LVAS after the first PET and before the second PET studies. The PET studies were carried out 67-104 days (mean \pm SD $82 \pm 19$ days) after the implantation of LVASs, and 26-106 days (mean \pm SD $56 \pm 44$ days) after AMS transplantation therapy. All subjects gave written informed consent according to a protocol approved by the Ethical Committee and Internal Review Board of Osaka University.

\section{PET Scan}

The PET scanner was a HEADTOME-V tomograph (SHIMADZU Corp., Kyoto, Japan). ${ }^{16}$ All data were acquired in $2 \mathrm{D}$ mode. Reconstructed images were obtained using a filtered back-projection algorithm with a Gaussian filter of $9 \mathrm{~mm}$ (full width at half maximum). The matrix and voxel sizes of reconstructed image were $128 \times 128 \times 63$ and $2.03 \times 2.03 \times 3.13 \mathrm{~mm}^{3}$, respectively. No scatter correction was applied to the image reconstruction.

Each subject was laid on the bed of the PET camera without any fixation of the body, and scanned at rest. A transmission scan was carried out first for correction of photon attenuation (20 minutes on the healthy volunteers, 10-15 minutes on the patients). A ${ }^{15} \mathrm{O}-\mathrm{CO}$ emission scan for blood pool imaging was initiated 8 minutes after inhalation of ${ }^{15} \mathrm{O}-\mathrm{CO}$ gas for 2 minutes (3.0-3.2 GBq). The ${ }^{15} \mathrm{O}$-water dynamic emission scan was then carried out following intravenous administration of ${ }^{15} \mathrm{O}$-water (1.1 GBq over 40 seconds) into the brachial vein, except for one patient who received the administration via right femoral vein. ${ }^{15} \mathrm{O}$-water scans were performed for 6 minutes, using 26 dynamic frames consisting of $12 \times 5 \mathrm{~s}$, $8 \times 15 \mathrm{~s}$, and $6 \times 30 \mathrm{~s}$. ${ }^{15} \mathrm{O}$-water scans were performed only once on eight healthy volunteers; two of the volunteers underwent ${ }^{15} \mathrm{O}$-water scans twice. Thus, a total of twelve ${ }^{15} \mathrm{O}$ water scans were carried out on the healthy volunteers. One patient underwent PET scans three times (before and after the implantation of LVAS, and after the cell transplantation therapy); the other two were scanned twice (before and after the cell transplantation therapy). Thus, a total of seven PET studies were carried out on the patients.

\section{Motion Detection and Correction}

Subject motion during cardiac ${ }^{15} \mathrm{O}$-water PET was detected using an optical motion-tracking device, POLARIS (Northern Digital Inc., Canada). This method for motion correction (MC) is based on a rigid body model, and performed on each sinogram of the dynamic frame to correct for interscan (IS) and inter-frame (IF) motions, as shown in Figure 1. The correction process was performed automatically, based on 


\section{Attenuation map}
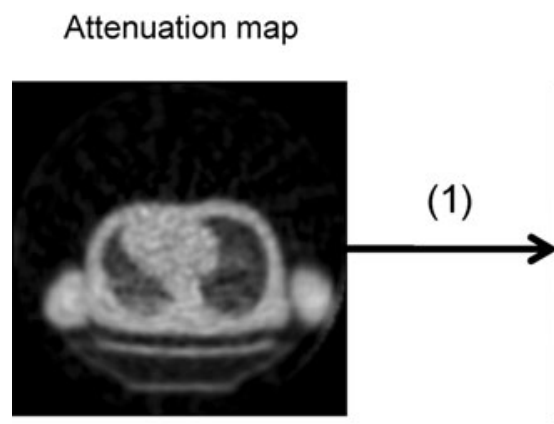

(1)

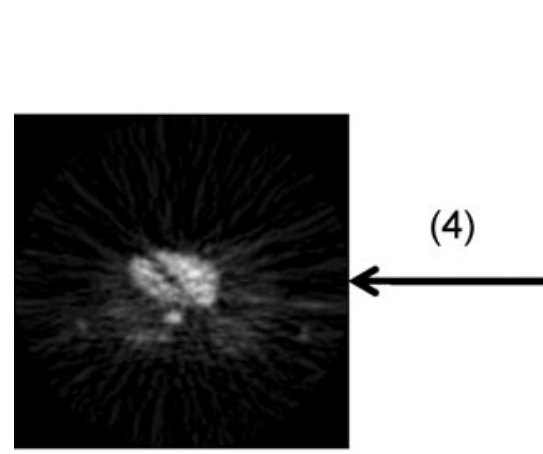

Corrected image
Realigned attenuation map

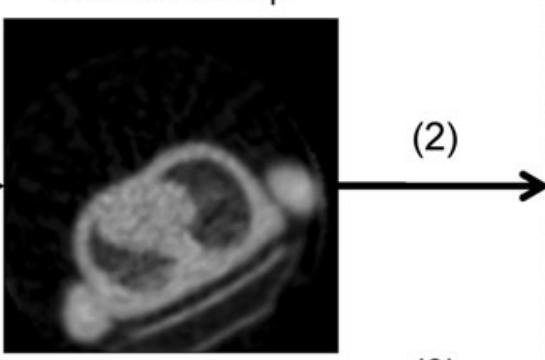

(3)
Attenuation sinogram

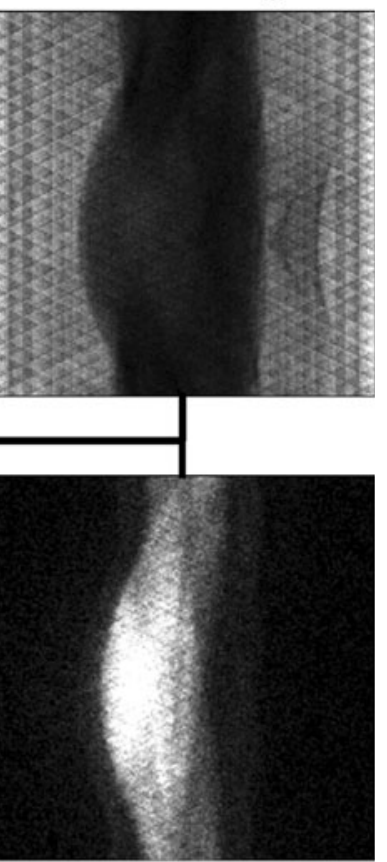

Emission sinogram

Figure 1. Schematic diagram of the correction for IS and IF motions.

user input consisting of subject locations measured by POLARIS, sinograms, and reconstruction parameters. In this manuscript, the IS motion denotes global motion between transmission and the first frame of ${ }^{15} \mathrm{O}$-water emission scan; the IF motion, which is in addition to the IS motion, denotes global motion between frames of the ${ }^{15} \mathrm{O}$-water scan. Methodological details regarding detection and correction of the motions are described in our previous studies. ${ }^{13,17,18}$

\section{Motion Classification}

Global movement during ${ }^{15} \mathrm{O}$-water scan was characterized as consisting of IS and IF motions. Accordingly, motion was classified as: (1) IS + IF, in which both IS and IF motions were present; (2) IS motion only; (3) IF motion only; and (4) neither type of movement was present (NE). The presence of each IS and IF motion was determined as described below. Using values $t_{x}(i), t_{y}(i)$, and $t_{z}(i)$ to represent translational movement in the $x, y$, and $z$, respectively, directions between the transmission and the $i$ th frame of the ${ }^{15} \mathrm{O}$-water scans, IS motion was considered significant if $\sqrt{t_{x}(1)^{2}+t_{y}(1)^{2}+t_{z}(1)^{2}}>4.0 \mathrm{~mm}$, where the value of $4.0 \mathrm{~mm}$ was the intrinsic spatial resolution of the PET scanner. ${ }^{16}$

IF motion was considered to be significant if $\max \{L(i), H(i)\}>4.0 \mathrm{~mm}$, where $L(i)$ represents the gradual movement of the subject during the scan, and $H(i)$ denotes the motion between adjacent frames. These two components of the IF motion can be expressed as

$$
\begin{aligned}
L(i) & =\sqrt{\sum_{w=x, y, z}\left[t_{w}(i)-t_{w}(1)\right]^{2}} \\
H(i) & =\sqrt{\sum_{w=x, y, z}\left[t_{w}(i)-t_{w}(i-1)\right]^{2}}
\end{aligned}
$$

\section{Motion Effect Evaluation}

To evaluate motion effects on perfusion and viability measurements, we estimated regional MBF and PTF values for nine myocardial segments (anterior, lateral, posterior, and septal wall regions at middle and basal levels, as well as apex) with and without MCs, as described in the following section. Percent differences in the estimated values between with and without MCs for the nine myocardial regions were statistically tested using a one-way ANOVA to find myocardial segments sensitive to global movement. The percent differences were employed owing to avoiding difference of physiological states cross the subjects. The percent difference was defined by $\% \Delta q(i)=100 \times\left|q_{\mathrm{W}}(i)-q_{\mathrm{Wo}}(i)\right| / q_{\mathrm{WO}}(i)$, where $q_{\mathrm{W}}(i)$ and $q_{\mathrm{WO}}(i)$ are $\mathrm{MBF}$ or PTF values, respectively, for the $i$ th myocardial segment with and without MCs. Absolute values of MBF and PTF with and without MCs for each group were also assessed using Bland-Altman analysis and a paired 2-tailed $t$ test. In the IS + IF motion group, to assess the effects of IS and IF motions, additional MBF and PTF estimations were performed on the data from ${ }^{15} \mathrm{O}$-water images corrected for IS motions (IS + IF - IS) and also on data corrected for IF motions (IS + IF - IF). The quantitative values obtained from IS + IF - IS data were considered to be affected by IF 
motion; likewise, values obtained from the IS + IF - IF data were considered to be affected by IS motion. The estimated values were compared to those without MCs using a paired 2 -tailed $t$ test. $P$ values $<.05$ were considered statistically significant. Data was expressed as mean $\pm 1 \mathrm{SD}$.

\section{MBF and PTF Estimation}

Regional MBF and PTF were obtained using the single tissue compartment model with correction for partial volume effects and spillover from the left ventricular cavity (LV)

$$
C(t)=\mathrm{MBF} \cdot \mathrm{PTF} \cdot C_{\mathrm{a}}(t) \otimes \exp \left(-\frac{\mathrm{MBF}}{p} t\right)+V_{\mathrm{a}} \cdot C_{\mathrm{a}}(t)
$$

where $C(t)$ represents the segment tissue time-activity curve (TTAC), $C_{\mathrm{a}}(t)$ represents the arterial time-activity curve, $p$ is the partition coefficient of water in the myocardial tissue $(0.91 \mathrm{~mL} / \mathrm{g})$, and $V_{\mathrm{a}}$ is the arterial blood volume and spillover fraction from LV. ${ }^{19}$ TTACs were generated for the nine myocardial segments using regions of interest (ROIs) selected within each ${ }^{15} \mathrm{O}$-water myocardial image with and without MCs. The myocardial image was obtained by subtraction of the early phase of the dynamic ${ }^{15} \mathrm{O}$-water image from the later phase. The midpoint between two phases was determined for each ${ }^{15} \mathrm{O}$-water image using the contrast between myocardial regions and $\mathrm{LV}$ cavities. To obtain $C_{\mathrm{a}}(t)$, a ROI was first drawn on a motion-corrected ${ }^{15} \mathrm{O}-\mathrm{CO}$ image, without regard to whether $\mathrm{MC}$ had been applied to the ${ }^{15} \mathrm{O}$-water image. The recovery coefficient of the $\mathrm{LV}$ was calculated from the ROI count on the ${ }^{15} \mathrm{O}-\mathrm{CO}$ image and the blood radioactivity concentration. Second, the ROI was transformed to the ${ }^{15} \mathrm{O}$ water image coordinate using a motion matrix that represents misalignment between transmission and the first dynamic frame of the ${ }^{15} \mathrm{O}$-water scans. This transformation was made to evaluate motion effects on the ${ }^{15} \mathrm{O}$-water scan, excluding effects of misalignment between ${ }^{15} \mathrm{O}-\mathrm{CO}$ and ${ }^{15} \mathrm{O}$-water scans. Using the transformed ROI, the LV TAC of the ${ }^{15} \mathrm{O}$-water image was calculated. Finally, $C_{\mathrm{a}}(t)$ was derived using the LV TAC and the overall TTAC was generated from nine myocardial segments according to the previous method. ${ }^{6}$ To demonstrate the influence of IS motion, the ROI-based approach was not used; instead, pixel-by-pixel MBF and PTF values were estimated for a representative normal scan. ${ }^{20}$ To denoise and smooth these parametric images, MBF and PTF were set to zero in voxels with $\mathrm{PTF}<0.3 \mathrm{~g} / \mathrm{mL}$ or $V_{\mathrm{a}}>0.8 \mathrm{~mL} / \mathrm{mL}$, and then filtered using a Gaussian filter of $14 \mathrm{~mm}$ (full width at half maximum). ${ }^{21}$ For normal scans in the group, improvement of homogeneity between myocardial segments by MC was also evaluated as $100 \times(1-$ $\mathrm{SD}_{\mathrm{W}} / \mathrm{SD}_{\mathrm{WO}}$ ), where $\mathrm{SD}_{\mathrm{W}}$ and $\mathrm{SD}_{\mathrm{WO}}$ were standard deviations of regional values with and without MCs, respectively.

\section{RESULTS}

Table 1 shows global movement during ${ }^{15} \mathrm{O}$-water scans, categorized by the presence of IS and IF motions. Thirteen scans ( 7 scans on healthy volunteers and 6 scans on patients) exhibited IS motions greater than $4.0 \mathrm{~mm}$, and 10 scans (5 scans on healthy volunteers and 5 scans on patients) exhibited IF motions greater than $4.0 \mathrm{~mm}$. Among these, 4 scans on healthy volunteers and 5 scans on patients showed both IS and IF motions.

There was no statistically significant difference between the ROI volumes of ${ }^{15} \mathrm{O}$-water images with and without MCs for IS, IF, and NE motion groups by paired 2-tailed $t$ tests, and between the volumes with and without MCs, IS + IF - IS, and IS + IF - IF motion for IS + IF motion group by a one-way ANOVA.

Table 2 lists MBF and PTF values with and without MCs categorized by the four types of movement that occurred during ${ }^{15} \mathrm{O}$-water studies, and average percent differences of MBF and PTF values over nine segments. Among 171 myocardial segments from all subjects, the fitting program failed to provide physiologically meaningful values in two segments (middle and basal septa for a healthy volunteer, in which IS + IF motion was detected) in the absence of MC. Those data were excluded from subsequent analysis. Among MBF values, significant changes by MCs were found in the IS + IF, IS + IF - IF, and IF motion groups: for $\mathrm{IS}+\mathrm{IF}$, from $0.845 \pm 0.366$ to $0.769 \pm 0.319 \mathrm{~mL} / \mathrm{min}-$ ute/g $(P<.05)$; for IS + IF - IF, from $0.845 \pm 0.366$ to $0.780 \pm 0.360 \mathrm{~mL} / \mathrm{minute} / \mathrm{g}(P<.05)$; and for $\mathrm{IF}$, from $0.854 \pm 0.179$ to $1.088 \pm 0.154 \mathrm{~mL} / \mathrm{minute} / \mathrm{g}$ $(P<.01)$. PTF values in the IS motion group changed significantly from $0.465 \pm 0.118$ to $0.504 \pm 0.087 \mathrm{~g} / \mathrm{mL}$ $(P<.05)$. This significant change is also shown in Figures 2 and 3, as differences between PTF values with and without MCs. In Figure 3, a data point indicated an averaged value for each scan.

Figure 4 illustrates the effect of IS motion of $5.0 \mathrm{~mm}$ on MBF and PTF of a normal subject from the IS motion group. The polar maps of normal PTF in Figure 4 demonstrate improvement of homogeneity in anterolateral regions by MC. Improvements of homogeneity of three normal scans in the group were $2.6 \% \pm 26.1 \%$ for $\mathrm{MBF}$ and $30.9 \% \pm 22.7 \%$ for PTF.

Table 1. Characteristics of motion during ${ }^{15} \mathrm{O}$ water scans

\begin{tabular}{lccc}
\hline $\begin{array}{c}\text { Motion } \\
\text { type }\end{array}$ & $\begin{array}{c}\text { Total } \\
\text { (normal) } \\
\text { scans }\end{array}$ & $\begin{array}{c}\text { IS } \\
\text { motion } \\
\text { (mm) }\end{array}$ & $\begin{array}{c}\text { IF } \\
\text { motion } \\
\text { (mm) }\end{array}$ \\
\hline IS + IF & $9(4)$ & $7.7 \pm 2.7$ & $11.0 \pm 4.0$ \\
IS & $4(3)$ & $5.4 \pm 1.0$ & $2.8 \pm 1.1$ \\
IF & $1(1)$ & 2.7 & 5.1 \\
NE & $5(4)$ & $2.5 \pm 0.7$ & $1.4 \pm 0.5$ \\
\hline
\end{tabular}


Table 2. The summary of MBF and PTF values with and without MCs

\begin{tabular}{lccc} 
Motion type & Without MC & With $\mathbf{M C}$ & Percent differen \\
\hline MBF $(\mathrm{mL} /$ minute/g) & & & \\
IS + IF & $0.845 \pm 0.366$ & $0.769 \pm 0.319^{*}$ & $33.8 \pm 61.5$ \\
IS + IF - IS & & $0.815 \pm 0.341$ & $21.2 \pm 38.5$ \\
IS + IF - IF & $0.855 \pm 0.343$ & $0.828 \pm 0.259$ & $25.1 \pm 41.4$ \\
IS & $0.854 \pm 0.179$ & $1.088 \pm 0.154^{\dagger}$ & $9.0 \pm 8.9$ \\
IF & $0.898 \pm 0.233$ & $0.909 \pm 0.221$ & $30.2 \pm 20.9$ \\
NE & & & $9.7 \pm 15.7$ \\
PTF (g/mL) & $0.476 \pm 0.133$ & $0.469 \pm 0.128$ & $13.9 \pm 10.9$ \\
IS + IF & & $0.471 \pm 0.135$ & $12.3 \pm 13.0$ \\
IS + IF - IS & & $0.490 \pm 0.119$ & $12.3 \pm 10.2$ \\
IS + IF - IF & $0.465 \pm 0.118$ & $0.504 \pm 0.087^{*}$ & $9.0 \pm 9.6$ \\
IS & $0.512 \pm 0.067$ & $0.483 \pm 0.068$ & $7.1 \pm 5.6$ \\
IF & $0.488 \pm 0.095$ & $0.496 \pm 0.100$ & $5.1 \pm 4.9$
\end{tabular}

$I S$, Inter-scan; $I F$, inter-frame.

$\mathrm{NE}$ denotes motion $\leq 4.0 \mathrm{~mm}$. IS + IF - IS and IS + IF - IF represent IS + IF motion groups with correction of IS and IF motions, respectively.

${ }^{*} P<.05,{ }^{\top} P<.01$ vs without MC.
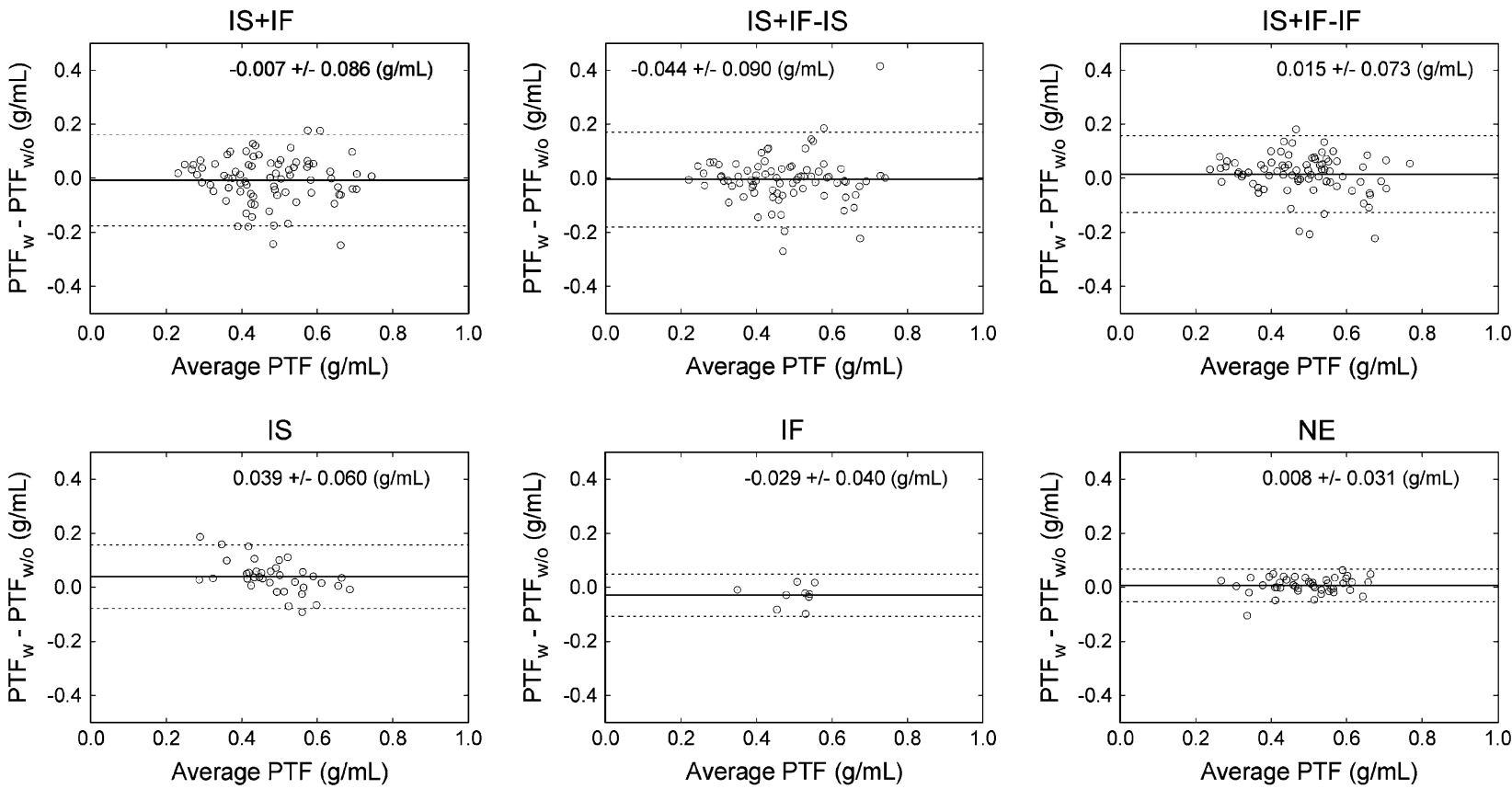

Figure 2. Differences between PTF values with and without the motion corrections. IS, Inter-scan motion; $I F$, inter-frame motion; $N E$, no significant motion. IS + IF - IS and IS + IF - IF are groups corrected for IS and IF motion in the IS + IF group, respectively.

Relative variability of regional MBF and PTF values due to global motion are shown in Figures 5 and 6, respectively. For MBF and PTF values in each motion group including the IS + IF - IS and IS + IF - IF groups, no significant difference between myocardial segments was observed in a one-way ANOVA (IF 

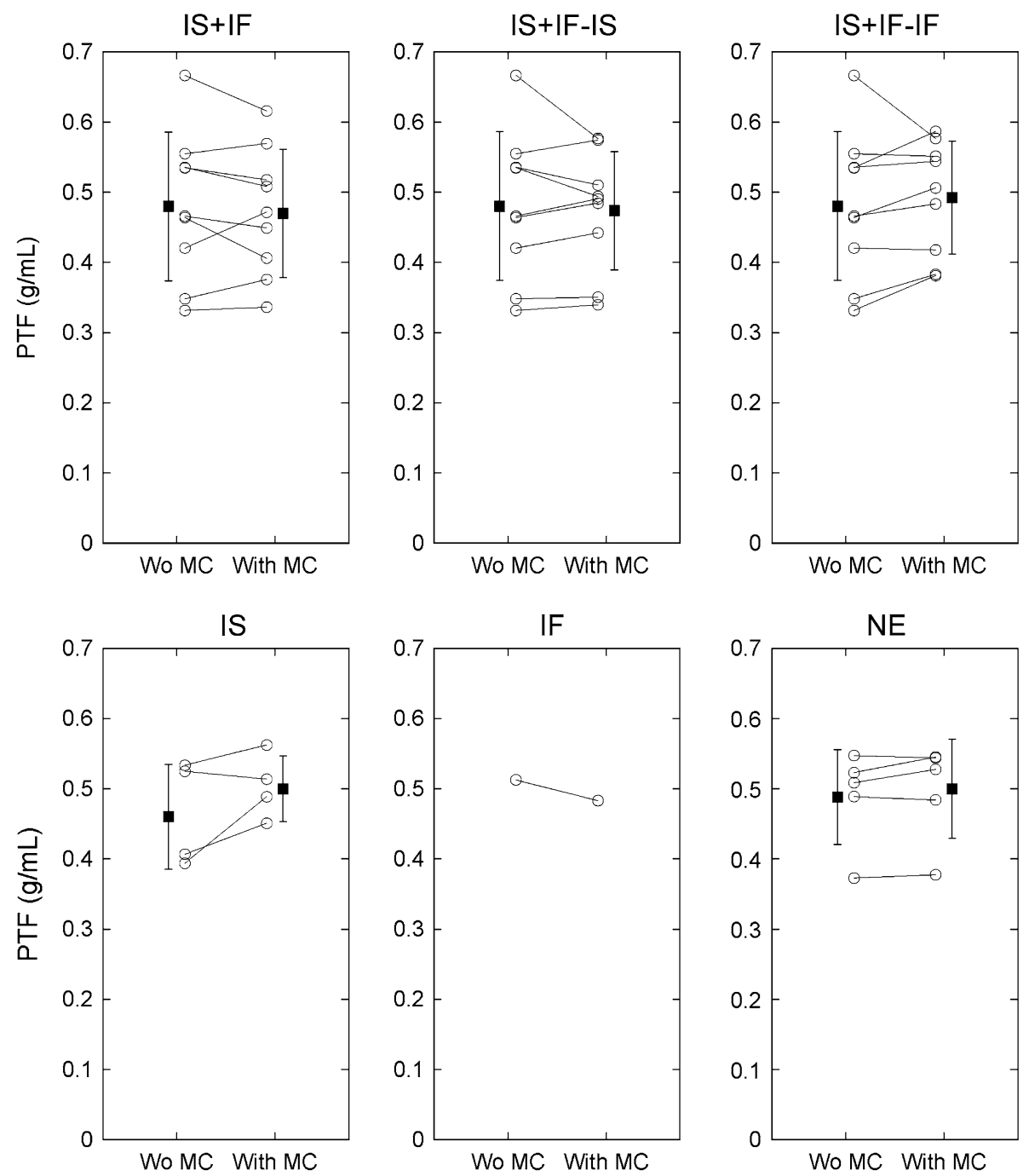

Figure 3. Changes in PTF values in the absolute scale by the MCs. $I S$, Inter-scan motion; $I F$, interframe motion; NE, no significant motion. IS + IF - IS and IS + IF - IF are groups corrected for IS and IF motion in the IS + IF group, respectively. A data point indicates an averaged value for each scan.

motion group was excluded from this analysis because one scan was assigned to this group. Data for IS + IF IS and IS + IF - IF group were not shown).

\section{DISCUSSION}

In this study, global movements of the subjects during ${ }^{15} \mathrm{O}$-water PET scans at rest were categorized as IS and IF motions. IS motion involves misalignment relative to the transmission scan, whereas IF motions involves changes between the dynamic frames during the ${ }^{15} \mathrm{O}$-water scan. After categorization, the effects of these motions on MBF and PTF measurements were evaluated. Consequently, it was demonstrated that
MBF values are affected by IF motion rather than IS motion.

Investigation of regional sensitivity to global movement resulted in no significant difference in regional MBF or PTF. This is because the direction and the magnitude of global movement varied for each subject; under- and over-estimation of MBF and PTF due to the motion could have occurred in any myocardial region. In this study, we utilized a one-way ANOVA to detect segmental differences. Due to small sample sizes, the ANOVA may fail to detect the differences. Correlation between segmental differences and the directions of global motions, especially IS motion, effects of which have been reported for misregistration between CT 


\section{$\mathrm{MBF}(\mathrm{mL} / \mathrm{min} / \mathrm{g})$}

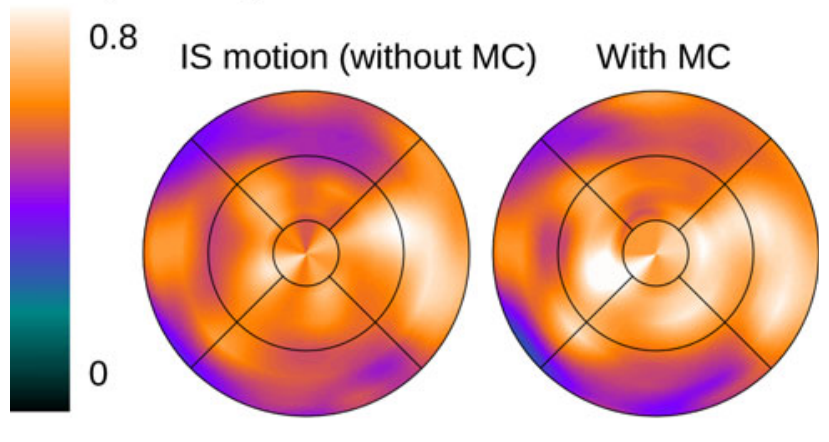

\section{PTF $(\mathrm{g} / \mathrm{mL})$}

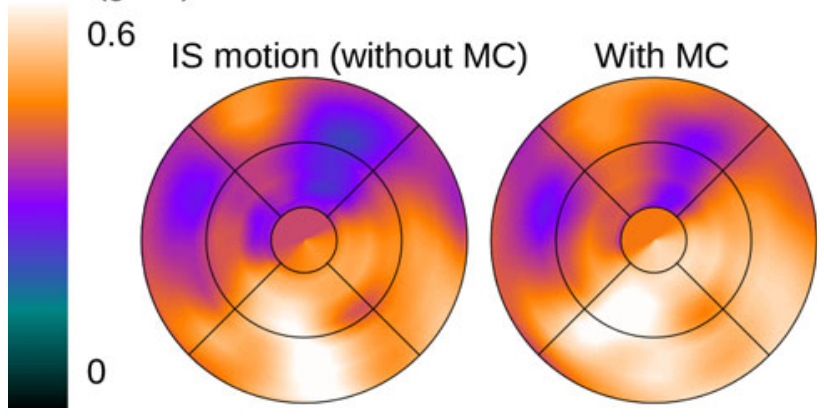

Figure 4. Effect of an IS motion of $5.0 \mathrm{~mm}$ on MBF and PTF of a normal subject in IS motion group.

attenuation map and emission image, ${ }^{22,23}$ could be observed in large population study.

MBF values changed significantly upon correction for IF motions in the IF, IS + IF, and IS + IF - IF motion groups. In contrast, correction of IS motion was considered to have little effect, based on the results from the IS + IF - IS and IS motion groups in Table 2. It was further demonstrated that IF motion, but not IS motion, was an important source of error in the MBF measurement. The significant but small changes in MBF of IF, IS + IF, and IS + IF - IF motion groups after MCs could be due to the relatively large number of healthy volunteer scans in each group (1 of 1 scan for IF, 4 of 9 scans for IS + IF, respectively), as shown in Table 1. Suppression of IF motion during the ${ }^{15} \mathrm{O}$-water scan is needed for accurate MBF measurement. For the IS motion groups, no significant change in MBF values and significant change in PTF values were observed by MCs (Figures 2, 3). For the normal scans in the group, the improvement of regional homogeneity was also observed in PTF rather than MBF. This is because the MBF values obtained using the kinetic model employed in this study were based on a clearance rate assessment of ${ }^{15} \mathrm{O}$-water rather than the uptake rate of the radiotracer. ${ }^{5}$ This is consistent with the findings of Lubberink et $\mathrm{al}^{21}{ }^{2}$ who reported that MBF values do not change even if attenuation correction is omitted; this correction only caused changes in the absolute scale of TTAC, which then caused changes in PTF values. IS motions were also detected in the IS + IF motion group. For the IS + IF - IS motion group, in which IS motions were corrected, no significant change in PTF values was observed after MCs. This might be because IS and IF motions affected the ${ }^{15} \mathrm{O}$-water images not subjected to MC in such a way as to cancel out errors in PTF measurements. PTF values were considered to be more affected than MBF values during the wash-in phase. However, in our study, IF motion had little effect on PTF values for the IS + IF - IF and IF groups, as shown in Table 2. The reasons for this discrepancy are follows: (1) to estimate the quantitative values, a nonlinear least squares method with same weights for all data points were applied to the data corrected for the physical decay. This fitting manner could be sensitive to radioactivity concentration in wash-out phase rather than wash-in phase. (2) Over-estimation in TTAC was introduced by contamination of the LV cavity count, using the ROI for TTAC superimposed on dynamic frames. However, the spillover correction could suppress the influence of IF motion. One of the advantages of ${ }^{15} \mathrm{O}$-water over other cardiac PET tracers is that perfusion and viability measurements can be obtained from a single PET scan with short duration. Because accurate PTF measurement enabled assessment of 

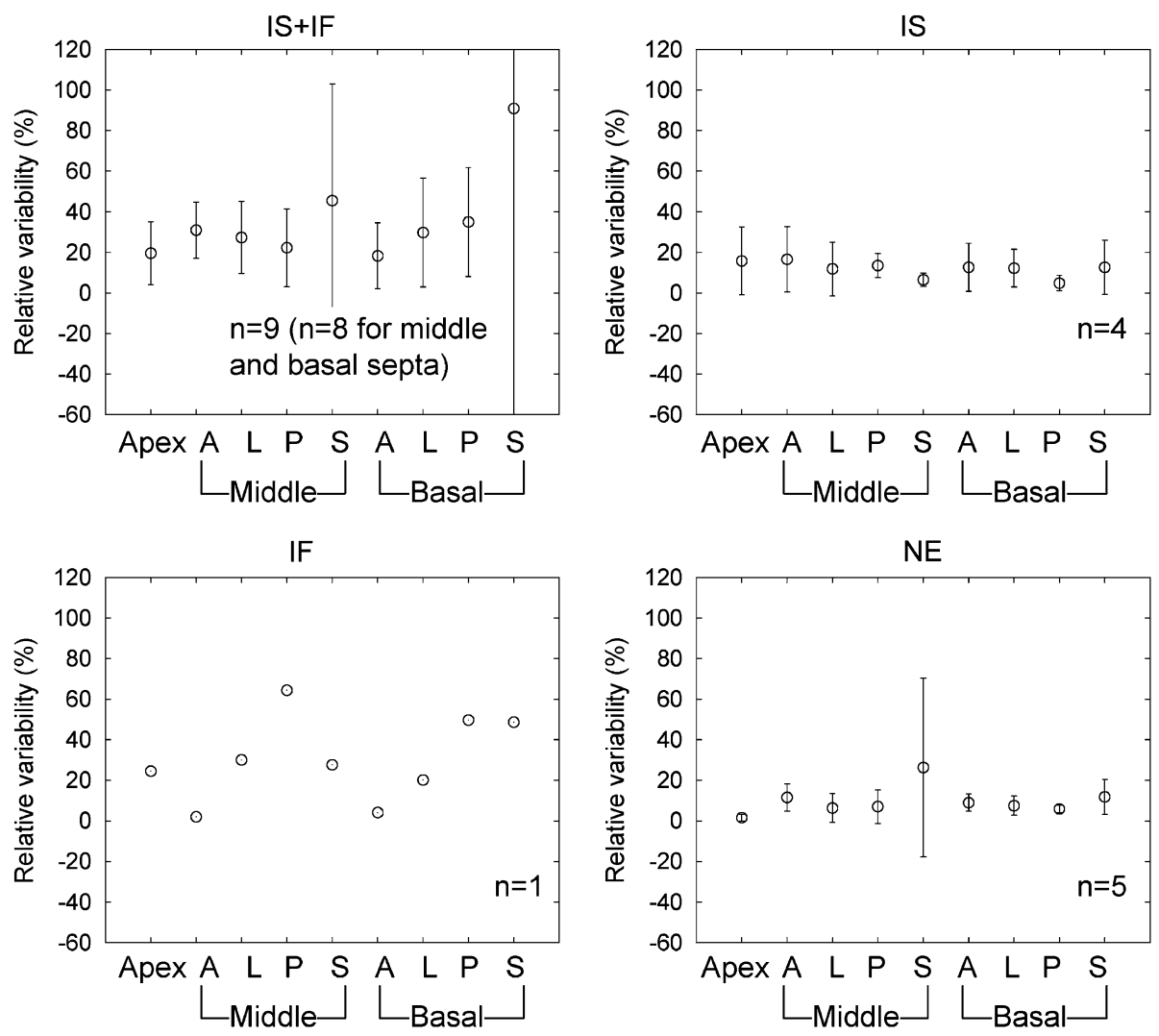

Figure 5. Relative variability of regional MBF due to effects of global motion. IS, Inter-scan motion; $I F$, inter-frame motion; NE represents no significant motion. IS + IF - IS and IS + IF IF are groups corrected for IS and IF motion in the IS + IF group, respectively.

myocardial viability, ${ }^{7-12}$ correction of IS motion, the effect of which was shown as the improvement of regional homogeneity of normal PTF in Figure 4, is considered to be important in the diagnosis of myocardial infarction, or evaluation of effects of cell transplantation therapies.

In this study, we performed ${ }^{15} \mathrm{O}-\mathrm{CO}$ scans to determine a recovery coefficient for each subject. ${ }^{5,6}$ ${ }^{15} \mathrm{O}$-water PET studies without ${ }^{15} \mathrm{O}-\mathrm{CO}$ scans have been reported in previous papers ${ }^{3,21,24}$; in these studies, the recovery coefficient could be fixed, or assumed to have a constant value. Omission of the ${ }^{15} \mathrm{O}-\mathrm{CO}$ scan shortened the examination time, and might reduce motion artefacts. However, we considered that the adequacy of using a fixed recovery coefficient is still an unresolved issue in ${ }^{15} \mathrm{O}$-water PET. We considered that the changes in $\mathrm{MBF}$ and PTF resulting from MCs were due to correction for global motion, and not due to variability of myocardial tissue ROIs drawn manually on ${ }^{15} \mathrm{O}$-water images. We employed nine segmented regions rather than 17-segment AHA standard model for suppressing relatively large noise level of ${ }^{15} \mathrm{O}$-water data. ${ }^{4}$ In addition, the volumes of the ROIs for each motion group before and after MCs were almost equal (not statistically significant). Furthermore, lower sensitivity of MBF values to variation in ROI size and shape was demonstrated by Iida et al. ${ }^{4}$ In this study, global motion might have occurred because subjects were scanned without any fixation. Although tight fixation prevents patient motion, such fixation could bring discomfort or pain to the patient; subsequent reaction to such pain could itself induce motion.

One limitation of this study is that all subjects were scanned at rest. When pharmacological or physiological stressors are administered, motion artefacts could be induced, as shown by Naum et $\mathrm{al}^{3}$ in the context of physiological stress conditions. Our finding that MBF was sensitive to IF motion rather than IS motion was considered to be valid for a stress study; IF motion might be source of severe error in MBF and CFR measurements. The rigid body model, which was used in this study, has been promoted for motion compensation not only in brain PET but also in cardiac PET. McCord et $\mathrm{al}^{1}$ employed the rigid body model to correct for misalignment during the transmission and ${ }^{18} \mathrm{~F}$-fluorodeoxyglucose $\left({ }^{18} \mathrm{~F}-\mathrm{FDG}\right)$ emission scans. Bacharach et $\mathrm{al}^{2}$ 

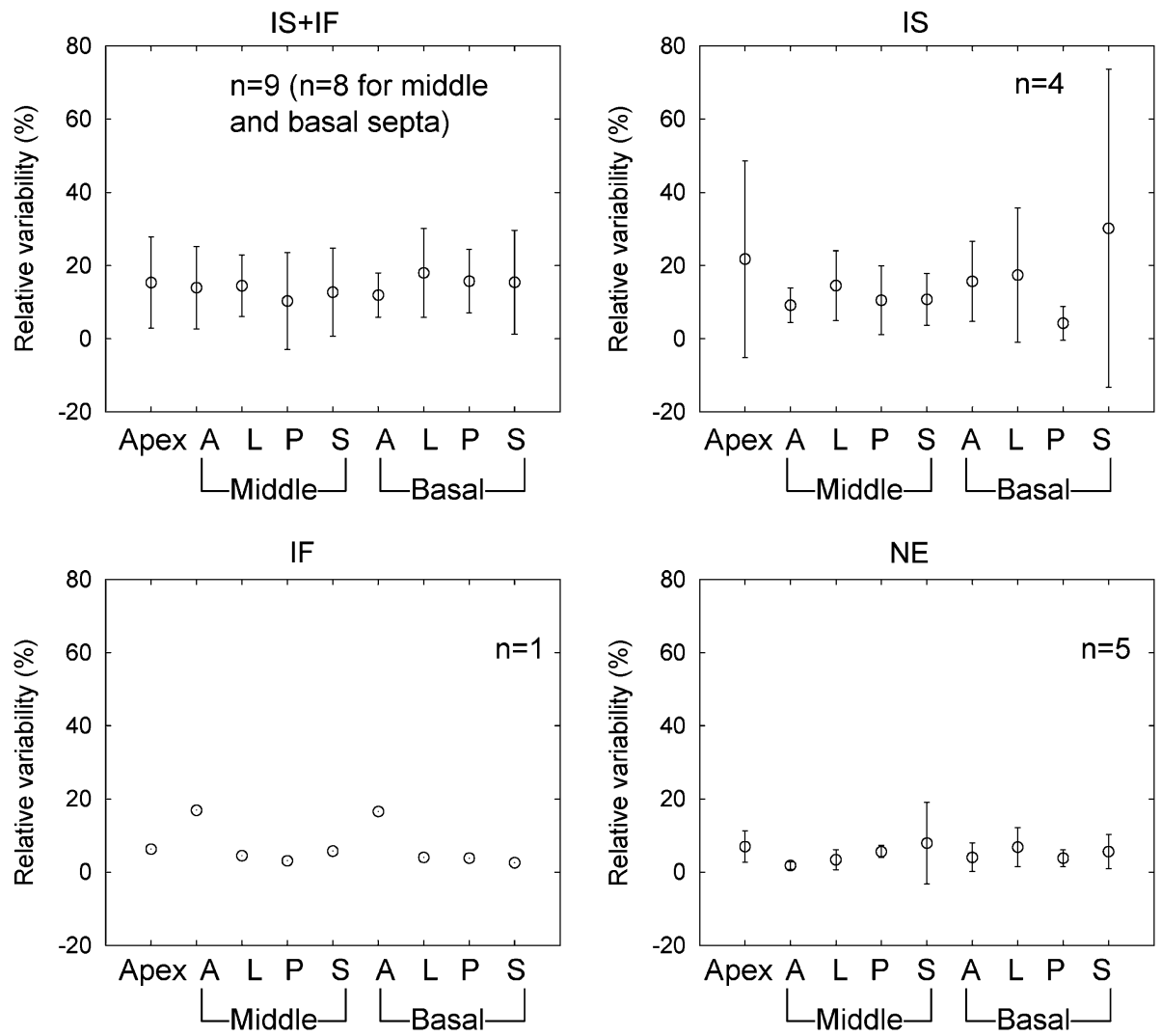

Figure 6. Relative variability of regional PTF due to effects of global motion. IS, Inter-scan motion; IF, inter-frame motion; NE represents no significant motion. IS + IF - IS and IS + IF IF are groups corrected for IS and IF motion in the IS + IF group, respectively.

also proposed a registration technique based on the rigid body model for ${ }^{18} \mathrm{~F}$-FDG emission images acquired on different days. The use of the rigid body model is considered to be valid for non-gated cardiac PET images because the images were smoothed spatially due to cardiac wall motion, and also averaged temporally over the duration of the dynamic frames. However, in stress studies, over-correction due to relatively large respiratory motions might be introduced by our system because the subject's motion was estimated by measurement of the location of a target attached on the thoracic surface. With this in mind, further work is necessary to evaluate the contribution of MC in combination with a respiratory gating technique.

The MC system was applied to clinical follow-up studies of the patients who received LVAS and cell transplantation therapy. The aim of this study was to investigate the effects of global movement on quantification of MBF and PTF values in a single ${ }^{15} \mathrm{O}$-water PET study under resting conditions. Although, we believed that the MC system could contribute to an accurate evaluation of regional perfusion and viability, the effects of LVAS implantation and AMS transplantation therapy on those patients is beyond the scope of the present study, and will be studied elsewhere.

\section{CONCLUSIONS}

This study demonstrated that IF motion during ${ }^{15} \mathrm{O}$ water scans under resting conditions could be the source of error in MBF measurement. Furthermore, estimated MBF values were less sensitive than PTF values to misalignment between the transmission and the ${ }^{15} \mathrm{O}$ water emission scans.

\section{Acknowledgments}

The authors are much indebted to the technical staff of the Department of Radiology, Osaka University Hospital, for their assistance. This study was supported by a Research Grant from the New Energy and Industrial Technology Development Organization (NEDO), Japan; a Grant for Translational Research from the Ministry of Health, Labour and Welfare (MHLW) of Japan; a Grant for Advanced Medical Technology from the Ministry of Health, Labour and Welfare (MHLW) of 
Japan; the Program for Promotion of Fundamental Studies in Health Science of the Organization for Pharmaceutical Safety and Research of Japan; and a Grant-in-Aid for Scientific Research from the Ministry of Education, Culture, Sports, Science and Technology (MEXT) of Japan

\section{Conflicts of interest}

The authors have no conflicts of interest to disclose.

\section{Open Access}

This article is distributed under the terms of the Creative Commons Attribution License which permits any use, distribution, and reproduction in any medium, provided the original author(s) and the source are credited.

\section{References}

1. McCord ME, Bacharach SL, Bonow RO, Dilsizian V, Cuocolo A, Freedman N. Misalignment between PET transmission and emission scans: Its effect on myocardial imaging. J Nucl Med 1992:33:1209-14.

2. Bacharach SL, Douglas MA, Carson RE, Kalkowski PJ, Freedman NM, Perrone-Filardi P, et al. Three-dimensional registration of cardiac positron emission tomography attenuation scans. J Nucl Med 1993;34:311-21.

3. Naum A, Laaksonen MS, Tuunanen H, Oikonen Va, Teräs M, Kemppainen J, et al. Motion detection and correction for dynamic (15)O-water myocardial perfusion PET studies. Eur J Nucl Med Mol Imaging 2005;32:1378-83.

4. Iida H, Kanno I, Takahashi A, Miura S, Murakami M, Takahashi $\mathrm{K}$, et al. Measurement of absolute myocardial blood flow with $\mathrm{H} 215 \mathrm{O}$ and dynamic positron-emission tomography. Strategy for quantification in relation to the partial-volume effect. Circulation 1988;78:104-15. (Erratum in: Circulation 1988;78:1078).

5. Iida H, Rhodes CG, de Silva R, Yamamoto Y, Araujo LI, Maseri A, et al. Myocardial tissue fraction-correction for partial volume effects and measure of tissue viability. J Nucl Med 1991;32:216975

6. Iida H, Rhodes CG, de Silva R, Araujo LI, Bloomfield PM, Lammertsma AA, et al. Use of the left ventricular time-activity curve as a noninvasive input function in dynamic oxygen-15-water positron emission tomography. J Nucl Med 1992;33:1669-77.

7. Yamamoto Y, de Silva R, Rhodes CG, Araujo LI, Iida H, Rechavia E, et al. A new strategy for the assessment of viable myocardium and regional myocardial blood flow using $15 \mathrm{O}$-water and dynamic positron emission tomography. Circulation 1992;86:167-78

8. de Silva R, Yamamoto Y, Rhodes CG, Iida H, Nihoyannopoulos P, Davies GJ, et al. Preoperative prediction of the outcome of coronary revascularization using positron emission tomography. Circulation 1992;86:1738-42.

9. Iida H, Rhodes CG, Araujo LI, Yamamoto Y, de Silva R, Maseri $\mathrm{A}$, et al. Noninvasive quantification of regional myocardial metabolic rate for oxygen by use of ${ }^{15} \mathrm{O}_{2}$ inhalation and positron emission tomography. Theory, error analysis, and application in humans. Circulation 1996;94:792-807.
10. Chareonthaitawee P, Kaufmann PA, Rimoldi O, Camici PG. Heterogeneity of resting and hyperemic myocardial blood flow in healthy humans. Cardiovasc Res 2001;50:151-61.

11. Knaapen P, Boellaard R, Götte MJ, van der Weerdt AP, Visser CA, Lammertsma AA, et al. The perfusable tissue index: A marker of myocardial viability. J Nucl Cardiol 2003;10:684-91.

12. Knaapen P, Boellaard R, Götte MJ, Dijkmans PA, van Campen LM, de Cock CC, et al. Perfusable tissue index as a potential marker of fibrosis in patients with idiopathic dilated cardiomyopathy. J Nucl Med 2004;45:1299-304.

13. Koshino K, Watabe H, Hasegawa S, Hayashi T, Hatazawa J, Iida $\mathrm{H}$. Development of motion correction technique for cardiac 15Owater PET study using an optical motion tracking system. Ann Nucl Med 2010;24:1-11.

14. Miyagawa S, Matsumiya G, Funatsu T, Yoshitatsu M, Sekiya N, Fukui S, et al. Combined autologous cellular cardiomyoplasty using skeletal myoblasts and bone marrow cells for human ischemic cardiomyopathy with left ventricular assist system implantation: Report of a case. Surg Today 2009;39:133-6.

15. Miyagawa S, Saito A, Sakaguchi T, Yoshikawa Y, Yamauchi T, Imanishi $\mathrm{Y}$, et al. Impaired myocardium regeneration with skeletal cell sheets-a preclinical trial for tissue-engineered regeneration therapy. Transplantation 2010;90:364-72.

16. Iida H, Miura S, Kanno I, Ogawa T, Uemura K. A new PET camera for noninvasive quantitation of physiological functional parametric images: HEADTOME-V-Dual. In: Myers R, Cunningham V, Bailey D, editors. Quantification of brain function using PET. London: Academic Press; 1996. p. 57-61.

17. Watabe H, Sato N, Kondoh Y, Fulton RR, Iida H. Correction of head movement using optical motion tracking system during PET study with rhesus monkey. In: Senda M, Kimura Y, Herscovitch P, editors. Brain imaging using PET. London: Academic Press; 2002. p. 1-8.

18. Woo SK, Watabe H, Yong C, Kim KM, Choon C, Bloomfield PM, et al. Sinogram-based motion correction of PET images using optical motion tracking system and list-mode data acquisition. IEEE Trans Nucl Sci 2004;51:782-8.

19. Katoh C, Morita K, Shiga T, Kubo N, Nakada K, Tamaki N, et al. Improvement of algorithm for quantification of regional myocardial blood flow using 15O-water with PET. J Nucl Med 2004;45:1908-16

20. Watabe H, Jino H, Kawachi N, Teramoto N, Hayashi T, Ohta Y, et al. Parametric imaging of myocardial blood flow with 15Owater and PET using the basis function method. J Nucl Med 2005;46:1219-24.

21. Lubberink M, Harms HJ, Halbmeijer R, de Haan S, Knaapen P, Lammertsma AA. Low-dose quantitative myocardial blood flow imaging using 15O-water and PET without attenuation correction. J Nucl Med 2010;51:575-80.

22. Goetze S, Brown TL, Lavely WC, Zhang Z, Bengel FM. Attenuation correction in myocardial perfusion SPECT/CT: Effects of misregistration and value of reregistration. $J$ Nucl Med 2007;48:1090-5.

23. Martinez-Möller A, Souvatzoglou M, Navab N, Schwaiger M, Nekolla SG. Artifacts from misaligned CT in cardiac perfusion PET/CT studies: Frequency, effects, and potential solutions. J Nucl Med 2007;48:188-93.

24. Nesterov SV, Han C, Mäki M, Kajander S, Naum AG, Helenius H, et al. Myocardial perfusion quantitation with 150-labelled water PET: High reproducibility of the new cardiac analysis software (Carimas). Eur J Nucl Med Mol Imaging 2009;36:1594-602. 